\title{
Skin Biosensing and Bioanalysis: What the Future Holds
}

\author{
Keng Wooi $\mathrm{Ng}^{1 *}$, S. Moein Moghimi ${ }^{1,2 *}$ \\ ${ }^{I}$ School of Pharmacy, Faculty of Medical Sciences, Newcastle University, King George VI Building, Newcastle \\ upon Tyne NE1 7RU, United Kingdom \\ ${ }_{2}^{2}$ Institute of Cellular Medicine, Newcastle University, Framlington Place, Newcastle upon Tyne NE2 4HH, \\ United Kingdom
}

Submitted: July 9, 2018

Accepted: July 26, 2018

Posted July 28, 2018

\section{Abstract}

Wearable skin biosensors have important applications in health monitoring, medical treatment and theranostics. There has been a rapid growth in the development of novel biosensing and bioanalytical techniques in recent years, much of it underpinned by recent advancements in nanotechnology. As the two related disciplines continue to co-evolve, we take a timely look at some notable developments in skin biosensing/bioanalysis, scan the horizon for emerging nanotechnologies, and discuss how they may influence the future of biosensing/bioanalysis in the skin.

\section{Keywords:}

Bioelectronics, Biosensors, Skin, Microneedles, Nanoneedles, Wearable devices

\section{Purpose and Rationale}

The human skin is the body's largest organ offering a convenient conduit both for delivering therapeutic agents into the body, and also for gathering information on homeostasis (e.g., cardiovascular activity, muscular activity and brain function through electrocardiograms. electromyograms and electroencephalograms). Furthermore, many diseases manifest as visible and sensual changes in the skin, such as altered skin colour, skin tone, emergence of lesions, bleeding or pain. Skin cancers, for example, are usually associated with visible skin lesions, and clinical diagnosis starts with physical examination of suspicious skin lesions. Importantly, such manifestation of signs and symptoms is not limited to local skin disorders but may offer information to physiological aberrations elsewhere in the body. A classic example is skin yellowing associated with jaundice, which may indicate a compromised liver function.
Our ability to detect changes in the skin, however, is limited by our natural senses, such as sight, touch, and the ability to sense pain. For instance, we have no intrinsic ability to detectlet alone accurately measure-molecular species or processes that underlie disease progression in a way that enables accurate diagnosis and timely treatment. Such an ability would obviously be beneficial because early and rapid diagnosis usually lead to therapeutic success, but it is often too late by the time more apparent signs such as bleeding emerge. In relation, the field of skin biosensing research has taken flight in the last couple of decades. The result is an array of skin biosensing technologies that augment our ability to detect and measure less apparent changes in the skin. Here, we outline some of those potentially disruptive technologies that may revolutionize biosensing and bioanalysis in the skin.

\footnotetext{
*Corresponding Authors: S. Moein Moghimi; Email: seyed.moghimi@ncl.ac.uk; Tel.: +44 1912082363

Keng Wooi Ng; Email: keng.ng@ncl.ac.uk; Tel.: +44 1912082343
} 


\section{Introduction and Discussion}

\section{Emerging technologies and future prospects}

There are two notable developments in wearable biosensing/bioanalytical techniques in the skin: microneedle devices and needleless devices containing skin surface electrodes.

Microneedles are microscopic needles, typically hundreds of microns long that are either solid or hollow. Such devices have a long history of development stretching back a few decades, especially in glucose monitoring. Most are designed to be a wearable skin patch where the protruding microneedles penetrate the skin barrier and insert into the superficial skin tissue. The technique is hailed minimally invasive because, although it results in physical disruption of the skin barrier, microneedle administration is typically painless and welltolerated. Most are based on interstitial fluid extraction through microfluidic circuits, coupled with enzymatic reactions (e.g., glucose oxidase) and real-time monitoring [1, 2]. Nonenzymatic glucose biosensors have also been developed [3]. Some microneedle devices incorporate a closed feedback loop to release therapeutic agents such as insulin on demand for self-actuated glucose control. Recently, microneedle biosensors have been developed for multiplex protein detection in the skin, including antibodies and cytokines $[4,5]$. Such devices employ antibody-antigen interactions to recognise specific target proteins in the interstitial fluid without extracting the interstitial fluid itself. Analyte detection is typically by established electrochemical, fluorescence or colorimetric techniques. Here, innovative paper-based analytical techniques provide economical alternatives suited for lowresource settings $[5,6]$.

On the other hand, skin surface electrodes are completely non-invasive, as they do not puncture the skin. They've been developed for sensing a range of physiological parameters, including glucose, pulse rate, $\mathrm{pH}$, and temperature. For instance, Lipani et al. [7] recently described a pixel array biosensor for non-invasive transdermal glucose monitoring. The device uses iontophoresis to extract glucose from interstitial fluids in the skin through hair follicles, followed by electrochemical detection with a graphene- based electrode. Another interesting development is a submicron-thick graphenebased biosensor designed to be laminated on the skin non-invasively like a temporary transfer tattoo [8]. The biosensor is transparent, stretchable and conforms well to the contours of the skin. The device successfully measures electrocardiograms, electroencephalograms, electromyograms, as well as skin temperature and hydration sensing. A wearable biosensor comprising a skin surface electrode for glucose monitoring in sweat, coupled with a microneedle-assisted metformin delivery system for blood glucose control has also been reported [9]. Such circuits may also be selfpowered by incorporating an internal power source [10-12].

For wearable biosensing devices that reside on the skin for continuous monitoring, user comfort is a prime consideration. This require flexible or stretchable materials, such as polyimide and polydimethylsiloxane with embedded electronic circuits $[13,14]$. There have been earnest attempts to make skin electronics similar in mechanical properties to the epidermis, to enhance conformity to skin contours, skin contact/adhesion, and user comfort [10]. These innovations have developed in tandem with an expanding repertoire of novel materials, such as lead zirconate titanate and semiconducting polymers (e.g., polyaniline) [14], although conventional approaches such as printed circuit board technologies have also been adapted for manufacturing stretchable electronic circuits intended as wearables [15].

Other innovative approaches include biosensing cells and 3-D printed biosensors. For example, Tastanova et al. [16] reported a cell-based biosensor for detecting asymptomatic cancers characterised by persistent mild hypercalcaemia. Cells engineered to express the human calciumsensing receptor were rewired to produce melanin in response to elevated $\mathrm{Ca}^{2+}$ concentrations. When implanted in the skin, the biosensor cells accumulate melanin under persistent hypercalcaemia, thus appearing darker than adjacent cells (similar to a tattoo). On the other hand, adaptive 3-D printing has enabled electronic circuits and cells to be printed directly on the skin, thus heralding a 
future of directly printed, personalised skin biosensors [17].

Nanotechnology has played an important role in these biosensing and bioanalytical applications and has the potential to do more. A range of electrochemical biosensors already utilise nanomaterials such as graphene, carbon nanotubes, metal nanoparticles, and nanowires in their electrodes [18-22]. Combinations of metallic nanowire networks and silk protein hydrogels are further improving biological compatibility of skin sensors and devices [18]. Moreover, quantum dots have been used to enhance target detection in immunoassays [23]. On the other hand, nanoneedles can measure mechanical changes within single cells in the skin beyond what microneedles can achieve [24], although as yet the technology is not wearable. Synthetic nano-complexes can be

\section{Conclusions}

The broad spectrum of exciting and emerging technologies in skin biosensing and bioanalysis, including "nanoneedles," sweat biosensors and sweat-stimulating sensors, are beginning to offer unprecedented opportunities for personalised and precision medicine at multiple fronts, yet many challenges still remain. Among the key determinants of success shared by many biosensing approaches and applications are biomarker discovery and validation, miniaturisation and conversion of hard circuits and transistor arrays to ultrathin flexible versions (e.g., stretchable electronics formed from liquid metal alloys), device specificity, durability, sensitivity, gas-permeability and of course "wearability" to follow the natural movement of the body. Safety issues also need addressing as sensors and devices should not induce inflammation on prolonged contact with the skin and block sweat glands [19]. Fabrication technologies should be scalable to drive eventual commercialisation of skin electronics and lab-on-skin devices. Here etching processes and inkjet printing are beginning to thrive such innovations [20]. A recent example is an intrinsically stretchable polymer transistor array with a device density of around 350 transistors per square centimetre [20]. Nevertheless, future developments in flexible, lightweight, and stretchable electronics that could be worn directly on the skin could offer unprecedented opportunities for monitoring of pathophysiological and vital sign signals during daily activity, exertion and sleep as well as medical treatment (e.g., pain management).

\section{Conflict of Interests}

The authors declare no conflicts of interest. For signed statements, please contact the journal office: editor@precisionnanomedicine.com

Quote As: Ng, KW, Moghimi, SM. Skin biosensing and bioanalysis: what the future holds. Prec. Nanomed. 2018;1(2):124-127. https://doi.org/10.33218/prnano1(2).180709.1

\section{References}

[1] P. M. Wang, M. Cornwell, and M. R. Prausnitz, "Minimally invasive extraction of dermal interstitial fluid for glucose monitoring using microneedles," Diabetes Technol. Ther., vol. 7, pp. 131141, Feb. 2005.

[2] L. M. Strambini et al., "Self-powered microneedle-based biosensors for pain-free highaccuracy measurement of glycaemia in interstitial fluid," Biosens. Bioelectron., vol. 66, pp. 162-168, Apr. 2015.

[3] S. R. Chinnadayyala, I. Park, and S. Cho, "Nonenzymatic determination of glucose at near neutral $\mathrm{pH}$ values based on the use of nafion and platinum black coated microneedle electrode array," Mikrochim. Acta, vol. 185, p. 250, Apr. 2018. 
[4] J. W. Coffey, S. R. Corrie, and M. A. F. Kendall, "Early circulating biomarker detection using a wearable microprojection array skin patch," Biomaterials, vol. 34, pp. 9572-9583, Dec. 2013.

[5] K. W. Ng, W. M. Lau, and A. C. Williams, "Towards pain-free diagnosis of skin diseases through multiplexed microneedles: biomarker extraction and detection using a highly sensitive blotting method," Drug Deliv. Transl. Res., vol. 5, pp. 387-396, Aug. 2015.

[6] D. Nicholas et al., "Rapid paper based colorimetric detection of glucose using a hollow microneedle device," Int. J. Pharm., vol. 547, pp. 244-249, Aug. 2018.

[7] L. Lipani et al., "Non-invasive, transdermal, path-selective and specific glucose monitoring via a graphene-based platform," Nat. Nanotechnol., vol. 13, pp. 504-511, Apr. 2018.

[8] S. K. Ameri et al., "Thinnest transparent epidermal sensor system based on graphene," in IEEE International Electron Devices Meeting (IEDM), pp. 18.4.1-18.4.4., 2016.

[9] H. Lee et al., "A graphene-based electrochemical device with thermoresponsive microneedles for diabetes monitoring and therapy," Nat. Nanotechnol., vol. 11, pp. 566-572, Jun. 2016.

[10] D.-H. Kim et al., "Epidermal electronics," Science, vol. 333, pp. 838-843, Aug. 2011.

[11] S. Xu et al., "Stretchable batteries with self-similar serpentine interconnects and integrated wireless recharging systems," Nat. Commun., vol. 4, p. 1543, Feb. 2013.

[12] K. Xie and B. Wei, "Materials and structures for stretchable energy storage and conversion devices," Adv. Mater., vol. 26, pp. 3592-3617, Jun. 2014.

[13] M. Gonzalez, F. Axisa, M. V. Bulcke, D. Brosteaux, B. Vandevelde, and J. Vanfleteren, "Design of metal interconnects for stretchable electronic circuits," Microelectron. Reliab., vol. 48, pp. 825-832, Jun. 2008.

[14] X. Wang, Z. Liu, and T. Zhang, "Flexible sensing electronics for wearable/attachable health monitoring," Small, vol. 13, p. 1602790, Jul. 2017.

[15] J. Vanfleteren et al., "Printed circuit board technology inspired stretchable circuits," MRS Bull., vol. 37, pp. 254-260, Mar. 2012.

[16] A. Tastanova et al., "Synthetic biology-based cellular biomedical tattoo for detection of hypercalcemia associated with cancer," Sci. Transl. Med., vol. 10, p. eaap8562, Apr. 2018.

[17] Z. Zhu et al., "3D printed functional and biological materials on moving free form surfaces," Adv. Mater., vol. 30, p. 1707495, Jun. 2018.

[18] M. Jo et al., "Protein-based electronic skin akin to biological tissues," ACS Nano, vol. 12, pp. 5637-5645, May 2018.

[19] A. Miyamoto et al., "Inflammation-free, gas-permeable, lightweight, stretchable on-skin electronics with nanomeshes," Nat. Nanotechnol., vol. 12, pp. 907-913, Sept. 2017.

[20] S. Wang et al., "Skin electronics from scalable fabrication of an intrinsically stretchable transistor array," Nature, vol. 555, pp. 83-88, March 2018.

[21] Y. Liu et al., "Lab-on-skin: a review of flexible and stretchable electronics for wearable health monitoring," ACS Nano, vol. 11, pp. 9614-9635, Sept. 2017.

[22] Y. Zhou et al., "Highly sensitive, self-powered and wearable electronic skin based on pressuresensitive nanofiber woven fabric sensor," Sci. Rep., vol. 7, p.12949, Sept. 2017.

[23] N. Hildebrandt, L. J. Charbonnière, M. Beck, R. F. Ziessel, and H.-G. Löhmannsröben, "Quantum dots as efficient energy acceptors in a time-resolved fluoroimmunoassay," Angew. Chem. Int. Ed., vol. 44, pp. 7612-7615, Nov. 2005.

[24] J. Zhang et al., "Molecular recognition using corona phase complexes made of synthetic polymers adsorbed on carbon nanotubes," Nat. Nanotechnol., vol. 8, pp. 959-968 Nov. 2013.

[25] S. Woo and P. W. K. Rothemund, "Programmable molecular recognition based on the geometry of DNA nanostructures," Nat. Chem., vol. 3, pp. 620-627, Aug. 2011.

[26] Z. Zhao et al., "Nanocaged enzymes with enhanced catalytic activity and increased stability against protease digestion," Nat. Commun., vol. 7, p. 10619, Feb. 2016.

[27] S. An et al., "Self-junctioned copper nanofiber transparent flexible conducting film via electrospinning and electroplating," Adv. Mater., vol. 28, pp. 7149-7154, Jun. 2016.

[28] H.-C. Kim, S.-M. Park, and W. D. Hinsberg, "Block copolymer based nanostructures: materials, processes, and applications to electronics," Chem. Rev., vol. 110, pp. 146-177, Jan. 2010.

[29] Sun Ke, Wei Teng-Sing, Ahn Bok Yeop, Seo Jung Yoon, Dillon Shen J., and Lewis Jennifer A., "3D printing of interdigitated Li-ion microbattery architectures," Adv. Mater., vol. 25, pp. 45394543, Jun. 2013. 